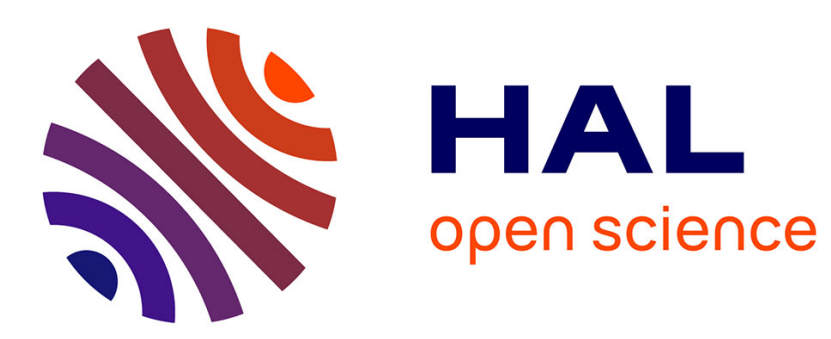

\title{
Influence of frictions on gait optimization of a biped robot with an anthropomorphic knee
}

\author{
Mathieu Hobon, Nafissa Lakbakbi Elyaaqoubi, Gabriel Abba
}

\section{To cite this version:}

Mathieu Hobon, Nafissa Lakbakbi Elyaaqoubi, Gabriel Abba. Influence of frictions on gait optimization of a biped robot with an anthropomorphic knee. Asian Control Conference, Jun 2013, Turkey. pp.1-6, 10.1109/ASCC.2013.6606365 . hal-00996655

\section{HAL Id: hal-00996655 https://hal.science/hal-00996655}

Submitted on 26 May 2014

HAL is a multi-disciplinary open access archive for the deposit and dissemination of scientific research documents, whether they are published or not. The documents may come from teaching and research institutions in France or abroad, or from public or private research centers.
L'archive ouverte pluridisciplinaire HAL, est destinée au dépôt et à la diffusion de documents scientifiques de niveau recherche, publiés ou non, émanant des établissements d'enseignement et de recherche français ou étrangers, des laboratoires publics ou privés. 


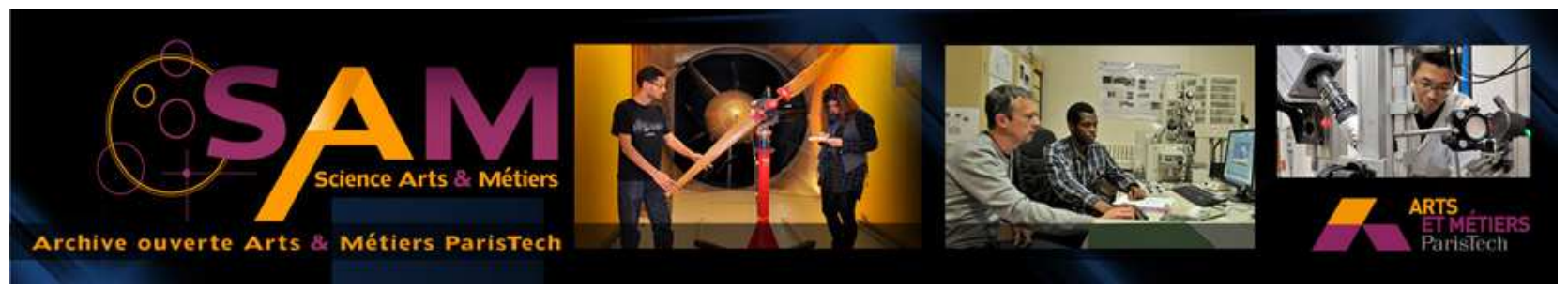

Science Arts \& Métiers (SAM)

is an open access repository that collects the work of Arts et Métiers ParisTech researchers and makes it freely available over the web where possible.

This is an author-deposited version published in: http://sam.ensam.eu

Handle ID: .http://hdl.handle.net/10985/8194

\section{To cite this version :}

Mathieu HOBON, Nafissa LAKBAKBI ELYAAQOUBI, Gabriel ABBA - Influence of frictions on gait optimization of a biped robot with an anthropomorphic knee - 2013 


\section{Influence of Frictions on Gait optimization of a Biped Robot with an Anthropomorphic Knee}

\author{
Mathieu Hobon \\ Design, Manufacturing and \\ Control Laboratory (LCFC) \\ Arts et Métiers ParisTech \\ 4 rue Augustin Fresnel \\ 57078 Metz, France
}

Email: mathieu.hobon@ensam.eu

\author{
Nafissa Lakbakbi Elyaaqoubi \\ Design, Manufacturing and \\ Control Laboratory (LCFC) \\ National Engineering College of Metz \\ 1 route d'Ars Laquenexy \\ 57035 Metz Cedex, France \\ Email: lakbakbi@enim.fr
}

\author{
Gabriel Abba \\ Design, Manufacturing and \\ Control Laboratory (LCFC) \\ National Engineering College of Metz \\ 1 route d'Ars Laquenexy \\ 57035 Metz Cedex, France \\ Email: gabriel.abba@ensam.eu
}

\begin{abstract}
This paper presents the energy consumption of a biped robot with a new modeled structure of knees which is called rolling knee (RK). The dynamic model, the actuators and the friction coefficients of the gear box are known. The optimal energy consumption can also be calculated. The first part of the paper is to validate the new kinematic knee on a biped robot by comparing the energy consumption during a walking step of the identical biped but with revolute joint knees . The cyclic gait is given by a succession of Single Support Phase (SSP) followed by an impact. The gait trajectories are parameterized by cubic spline functions. The energetic criterion is minimized through optimization while using the simplex algorithm and Lagrange penalty functions to meet the constraints of stability and deflection of the mobile foot. An analysis of the friction coefficients is done by simulation to compare the human characteristics to the robot with RK. The simulation results show an energy consumption reduction through the biped with rolling knee configuration. The influence of friction coefficients shows the energy consumption of biped robot is close to that of the human.
\end{abstract}

\section{INTRODUCTION}

\section{A. Design improvement}

The research on technology improvement in the field of biped and humanoid robots are numerous which focuses on different areas in mechanical structures, kinematic, control, human robot interaction and so on. The current research work concentrates on the following main aspects:

- The selection of the best kinematic structures of the knee joint [1],[2],[3]

- The improvement of gait support trajectories used by some control strategy [4], [5], [6]

These two parts contribute to improve the robot energetic efficiency during the gait and also the range of travel of the robot without recharging its battery, named in brief biped autonomy.

On a majority of biped prototype, the knee joints are classically realized by revolute joints. Biomechanical studies talk about the human knees that relate the movement of this articulation which is a combination of a rotation and a translation. [1] proposes knee structures combining these movements with a cross four-bar linkage. The simulations show less energy usage through this solution comparing to conventional revolute joint knees. Another knee mechanisms designed by [2] uses a singularity of the mechanism to save the energy. When the mobile leg is stretched at the end of the step, the knee is locked by the singularity and does not consume energy during the next stance phase. The energy consumption also decreases during the gait. The design of the knee mechanism in the LARP project in [7] is coming from the studies on prosthetic knees and consist of a structure with two cylindrical surfaces in contact. [3] proposes simulations with the same design for the knees. The structure is called rolling knee. It adapts on a biped with anthropomorphic model and is compared to a similar configuration with revolute joint knees. The results of this study show that the robot with rolling knees has allowed minimizing the sthenic criterion during walking for the same forward speed. Now, we have already done the same study on another biped model for which all characteristic of the actuators and the gear box are known. So, we want to know what is the energy consumption of the biped robot.

The gait is described by a succession of contacts between feet and the ground. The important issue is to keep the equilibrium of the biped during this progression. Some control strategies need stable reference trajectories to ensure the gait of the robot. The parametrization of the trajectories can be done by polynomial functions [8], Bézier functions [6], [9], Fourier series functions [10] or cubic splines [1]. Here, we use the cubic splines to parameterize the trajectories. In the optimization problem, the discussion is about the criteria. In [3], we use the sthenic criterion to compare our robot to a conventional revolute joint robot and this criterion permits the actuator selection. In this work, an energy criterion is used to calculate the energy consumption of two bipeds during the gait.

All works cited before use parametric gait and so transform the optimization problem to a parametric optimization problem. The choice of the algorithms to solve these problems is difficult. In this paper, the Nelder-Mead simplex algorithm [11] is used which allows to obtain a rapid convergence and to avoid local minima.

The last point of the discussion is about the coefficient of friction of the actuators. The biological studies show low friction on the human knee articulation. The friction is reduced with the viscosity of the lubrication, called synovial fluid, between the femur and the tibia. [12] relates the coefficient of friction of the articulation at 0.001. [13] studies compliant layer knee for prostheses composed by polyurethane for the 
tibial part and metal for the femoral part. The investigation shows the low friction factor included between 0.001 and 0.015. In general, the global Coulomb friction coefficient in the gear box is between of 0.08 to 0.1 . Our study concentrates on the importance of the coefficient of friction on the energy consumption, also on the autonomy of the biped. We calculate the estimated cost of the gait $\left(c_{e t}\right)$ defined in [14], [15] and compare our results to human and other robots.

\section{B. Contributions and outline}

This paper highlights the importance of mechanical friction in the energy performance of a biped robot. The friction influence also more the biped autonomy as the kinematic structure of the knee joint. In [3], the authors have proposed optimal trajectories in regards of a sthenic criterion. These trajectories minimize also the Joule losses in the motor. The results allow the selection of the motor and transmissions for each joint of the robot. We now present the next step in the robot design. The criterion is modified to take into account all losses. The friction model is certainly simplified, but enough just to show its importance [16], [17]. The rolling knee structure lends itself easier to control by linear motor. The paper clearly shows that the performance of the biped robot can approach that of humans at the price of a particular care to the mechanical design of the robot.

In section II, the models of the biped with the revolute joint knees and the anthropomorphic structure are established. The parametric gait formulation is discussed in the section III. The section IV introduces the energy optimization problem. Simulations and results are presented in section $\mathrm{V}$ to demonstrate that the friction influence more the energy criterion than the cinematic structure of the knee. Finally, section VI presents the conclusion and perspectives.

\section{MODEL}

\section{A. Biped Model}

This study is focused on the cyclic walk of the biped in the sagittal plane. The considered biped is composed of seven rigid bodies with two feet, two shins, two thighs and one trunk. The biped is all actuated by six actuators. For the study, we define two configurations. Both have revolute joints placed at hips and ankles level, but different knee joints. One with revolute joints on the knees named conventional configuration or CK Robot, and the second with rolling knees, named RK Robot. Here, we focus on the new knee structure. Fig. 1 shows the configurations with rolling knee. The rolling knee consists of a movement of two cylindrical surfaces rolling without sliding. The two surfaces are the terminal surface of the femur and the tibia. The reference frame is $\Re_{0}=\left(O_{0}, \overrightarrow{x_{0}}, \overrightarrow{y_{0}}, \overrightarrow{z_{0}}\right) . O_{0}$ is defined by the projection of the point $A_{1}$ on the ground. The direction of the walk is according to $\overrightarrow{x_{0}}$ and $\overrightarrow{z_{0}}$ the unit vector perpendicular to the ground. The orientation of the links are defined by the absolute angles $q_{i},\{i=0 \ldots 6\}$ referenced by the vertical (see Fig. 1), the speed vector $\dot{q}_{i},\{i=0 \ldots 6\}$ and the vector of articular torques $\Gamma=\left[\Gamma_{1} \ldots \Gamma_{6}\right]^{\mathbf{T}}$ which represents the torques placed on the hips, the knees and the ankles. Fig. 2 shows the details of the knee for the second configuration. The contact between the femur and the tibia is maintained with a bar on $C_{1}$ and $C_{2}$ of length $r_{1}+r_{2} . r_{1}$ and $r_{2}$ are respectively the

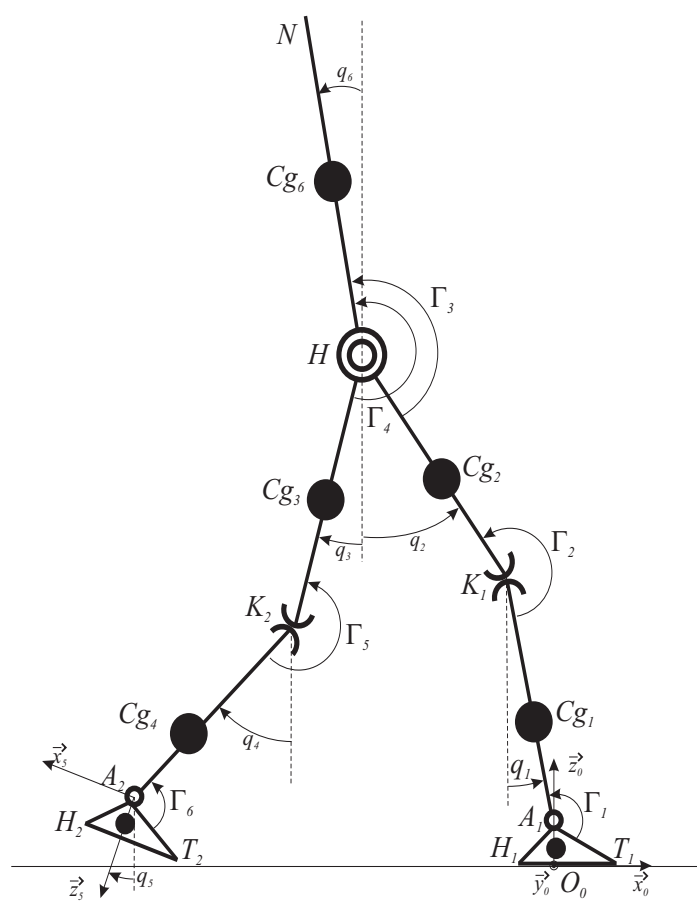

Fig. 1. Biped robot with rolling joint knees

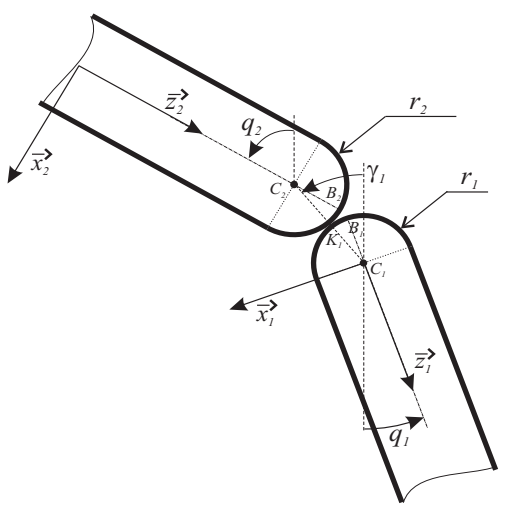

Fig. 2. Rolling knee of one leg

distance $B_{1} C_{1}$ and $B_{2} C_{2}$. With the rotation without sliding, we can write (1) and find the relation of the angle $\gamma_{1}$ which shows the coupling between the angles $q_{1}$ and $q_{2}$ for the support leg in (2). Similarly for the mobile leg, $\gamma_{2}$ is the coupling angle between $q_{3}$ and $q_{4}$ in (3).

$$
\begin{gathered}
\widehat{B_{1} K_{1}}=\widehat{B_{2} K_{1}} \\
\gamma_{1}=\frac{r_{1} q_{1}+r_{2} q_{2}}{r_{1}+r_{2}} \\
\gamma_{2}=\frac{r_{1} q_{4}+r_{2} q_{3}}{r_{1}+r_{2}}
\end{gathered}
$$

The dynamic model's parameters are the length $l_{i}$ of the links for the classic robot configuration, $i=\{0 \ldots 6\}$, the position of the center of mass $C_{g i}$, the masses $m_{i}$, the moments of inertia $I_{i}$ of each bodies $C_{i}$ around the $\overrightarrow{y_{0}}$ axis at $C_{g i}$. To compare the two configurations, we consider that the hip positions of the biped on vertical stance are equal in 
both cases. This assumption gives us $l_{1}^{\prime}=A_{1} C_{1}=l_{1}-r_{1}$ and $l_{2}^{\prime}=H C_{2}=l_{2}-r_{2}$.

The coordinates of the hip, the heel and the toes for the conventional biped configuration are:

$$
\begin{aligned}
x_{H}= & -l_{2} \sin q_{2}-l_{1} \sin q_{1} \\
z_{H}= & l_{2} \cos q_{2}+l_{1} \cos q_{1}+h_{p} \\
x_{H_{2}}= & x_{H}+l_{1} \sin q_{4}+l_{2} \sin q_{3} \\
& -l_{p} \cos q_{5}+h_{p} \sin q_{5} \\
z_{H_{2}}= & z_{H}-l_{1} \cos q_{4}-l_{2} \cos q_{3} \\
& -l_{p} \sin q_{5}-h_{p} \cos q_{5} \\
x_{T_{2}}= & x_{H}+l_{1} \sin q_{4}+l_{2} \sin q_{3} \\
& -\left(l_{p}-L_{p}\right) \cos q_{5}+h_{p} \sin q_{5} \\
z_{T_{2}}= & z_{H}-l_{1} \cos q_{4}-l_{2} \cos q_{3} \\
& -\left(l_{p}-L_{p}\right) \sin q_{5}-h_{p} \cos q_{5}
\end{aligned}
$$

The coordinates of the hip, the heel and the toes for the rolling knee configuration are:

$$
\begin{aligned}
x_{H}^{\prime}= & -l_{2}^{\prime} \sin q_{2}-\left(r_{1}+r_{2}\right) \sin \gamma_{1}-l_{1}^{\prime} \sin q_{1} \\
z_{H}^{\prime}= & l_{2}^{\prime} \cos q_{2}+\left(r_{1}+r_{2}\right) \cos \gamma_{1}+l_{1}^{\prime} \cos q_{1}+h_{p} \\
x_{H_{2}}^{\prime}= & x_{H}^{\prime}+l_{2}^{\prime} \sin q_{3}+l \sin \gamma_{2}+l_{1}^{\prime} \sin q_{4} \\
& -l_{p} \cos \left(q_{5}\right)+h_{p} \sin \left(q_{5}\right) \\
z_{H_{2}}^{\prime}= & z_{H}^{\prime}-l_{2}^{\prime} \cos q_{3}-l \cos \gamma_{2}-l_{1}^{\prime} \cos q_{4} \\
& -l_{p} \sin \left(q_{5}\right)-h_{p} \cos \left(q_{5}\right) \\
x_{T_{2}}^{\prime}= & x_{H}^{\prime}+l_{2}^{\prime} \sin q_{3}+l \sin \gamma_{2}+l_{1}^{\prime} \sin q_{4} \\
& -\left(l_{p}-L_{p}\right) \cos \left(q_{5}\right)+h_{p} \sin \left(q_{5}\right) \\
z_{T_{2}=}^{\prime} & z_{H}^{\prime}-l_{2}^{\prime} \cos q_{3}-l \cos \gamma_{2}-l_{1}^{\prime} \cos q_{4} \\
& -\left(l_{p}-L_{p}\right) \sin \left(q_{5}\right)-h_{p} \cos \left(q_{5}\right)
\end{aligned}
$$

\section{B. Dynamic Model}

In this work, we consider only the walking gait defined with Simple Support Phases (SSP) followed by an impact between the mobile foot and the ground. The impact produces the instantaneous exchange of support leg during the gait. The dynamic model for the SSP is assumed with the left leg on support. Considering the gait like periodic with a permutation of the legs at the impact, the study focuses on one step beginning with the impact. The dynamic and impact models are described as follow:

1) Dynamic model during the SSP: The Lagrange equations are used to determine the inverse dynamic model. Details of the modeling are mentioned in [18] and [19]. Posing $q=\left[q_{i}\right], i \in[0 \ldots 6]$, we express the state vector for the conventional configuration $X_{e}=\left[q, x_{H}, z_{H}\right]^{\mathbf{T}}$ of dimension $9 \times 1$. Respectively, the state vector of the rolling knee configuration is $X_{e}=\left[q, x_{H}^{\prime}, z_{H}^{\prime}\right]^{\mathbf{T}}$ of dimension $9 \times 1$ too. The relation between the torque vector $\Gamma_{m}$ of the actuator and the joint torque $\Gamma$ vector is expressed by:

$$
\Gamma=\Gamma_{m}-F v \dot{\theta}-C \operatorname{sign}(\dot{\theta})
$$

with $\dot{\theta}=\left[\dot{q}_{1}-\dot{q}_{0}, \dot{q}_{2}-\dot{q}_{1}, \dot{q}_{6}-\dot{q}_{2}, \dot{q}_{6}-\dot{q}_{3}, \dot{q}_{3}-\dot{q}_{4}, \dot{q}_{4}-\dot{q}_{5}\right]^{T}$, $F v=\operatorname{diag}\left(f v_{1}, f v_{2}, f v_{3}, f v_{3}, f v_{2}, f v_{1}\right)$ and $C s=$ $\operatorname{diag}\left(c s_{1}, c s_{2}, c s_{3}, c s_{3}, c s_{2}, c s_{1}\right)$ the matrices of viscosity and Coulomb friction. The inverse dynamic equation can be written as:

$$
\left[\begin{array}{ll}
B & \left.A_{c_{L}}\left(X_{e}\right)^{T}\right]\left[\Gamma F_{L}\right.
\end{array}\right]^{T}=D\left(X_{e}\right) \ddot{X}_{e}+H\left(\dot{X}_{e}, X_{e}\right)+Q\left(X_{e}\right)
$$

where $D\left(X_{e}\right)$ represents the inertia matrix $9 \times 9, H\left(\dot{X}_{e}, X_{e}\right)$ is the vector of Coriolis and centrifugal effects $9 \times 1, Q\left(X_{e}\right)$ is the vector of torques and forces due to the gravity $9 \times 1, B$ is the control matrix $9 \times 6$ and $A_{c_{L}}\left(X_{e}\right)$ is the Jacobian matrix $3 \times 9$ of the foot on support. The second derivative of the eq. (4) and (5) or the eq. (10) and (11) gives the expression of $\ddot{x}_{H}$ and $\ddot{z}_{H}$ respectively $\ddot{x}_{H}^{\prime}$ and $\ddot{z}_{H}^{\prime}$ needed for the determination of the state vector. These results can be expressed:

$$
A_{c L}\left(X_{e}\right)^{T} \ddot{X}_{e}+H_{c L}\left(X_{e}\right)=0
$$

with the evolution of the vector $X_{e}$ satisfying (18). The torque $\Gamma$ and external forces $F_{L}=\left[F_{x}, F_{z}, C_{y}\right]^{T}$ on the support stance ankle are calculated with (17) at any instant of the gait.

2) Impact model: During the gait, the left foot and then the right foot alternatively touch the ground with a non-zero speed. This is the impact phase. The impact phase separates two SSP. The impact phase between two rigid bodies, the foot and the ground, produces a mechanical energy dissipation phenomena [20]. We suppose that the restitution coefficient is equal to zero. This assumption ensures that we have no rebounds of the foot after the impact. The proposed model is given by:

$$
\begin{aligned}
D\left(X_{e}\right)\left(\dot{X}_{e}^{+}-\dot{X}_{e}^{-}\right) & =A_{c_{L}}^{T} I_{R} \\
A_{c_{L}}\left(X_{e}\right) \dot{X}_{e}^{+} & =0 \\
\Delta_{i}=D^{-1} A_{c_{L}}^{T} I_{R} & =\left[\Delta_{0}, \Delta_{1}, \Delta_{2}, \Delta_{3}, \Delta_{4}, \Delta_{5}, \Delta_{T}\right]^{T}
\end{aligned}
$$

This model is used to find the speed vector after the impact $\dot{X}_{e}{ }^{+}$from the configuration $X_{e}$ in relation with the speed vector before impact $\dot{X}_{e}{ }^{-} . \Delta_{i}$ is the difference between the speed vector after and before the impact. It will be used to define the initial conditions of the trajectories in the following section. This model also gives the impact screw on the foot $I_{R}=\left[I_{F x}, I_{F z}, C_{y}\right]^{T}$ and the torque applied on the ankle.

\section{GAIT REFERENCE PARAMETRIC TRAJECTORIES}

Now, the gait is defined by the evolution of angular coordinates of the bodies with respect to time. Our study is focused on the cubic spline trajectories. We express in this section the mathematical functions and we explain the reduction of parameters with the condition of cyclicity and impact. The angular coordinates $q_{i}$ with $i=\{0 \ldots 6\}$ are parameterized by a cubic spline function also, used in [1], [21]. To simplify the definition of the trajectories, the time $t$ is normalized to the dimensionless time variable $t_{n}=t / T$ with $T$ the step period. The gait can be described by: at $t_{n}=0$, the left foot is fixed on the ground and the right foot is behind the trunk. At $t_{n}=1$, the right foot has an advance of a distance $\frac{d}{2}$ and it is in front of the trunk.

\section{A. Cubic Spline Function}

In this case, the trajectories are defined by two cubic spline functions. Each function is parameterized for a half-period. The knot vector has three knots so we define $t_{k}=[0,0.5,1]$. 
In neighborhoods of $t_{i} \in t_{k}, i=[0,2]$, the spline function has the smoothness $\mathscr{C}^{1}$. We suppose that at the time $t_{1}=0.5$, the second derivative is continuous. Also, for $t_{0}=0$ and $t_{2}=1$, the impact imposes a discontinuity on the velocities.

The expression of the cubic spline function is:

$$
\begin{aligned}
& 0 \leq t_{n} \leq \frac{1}{2} \rightarrow f_{q_{i}}\left(t_{n}\right)=\sum_{j=0}^{3} a_{i_{j}} t_{n}^{j} \\
& \frac{1}{2} \leq t_{n} \leq 1 \rightarrow f_{q_{i}}^{\prime}\left(t_{n}\right)=\sum_{j=0}^{3} b_{i_{j}}\left(1-t_{n}\right)^{j}
\end{aligned}
$$

where $a_{j}$ and $b_{j}$ are the eight parameters expressed for each angle. Supposing $k=\frac{1}{T}$, the velocities and accelerations are obtained by derivation:

$$
\begin{aligned}
& \dot{q}_{i}(t)=k \frac{d f_{q_{i}}}{d t_{n}} \\
& \ddot{q}_{i}(t)=k^{2} \frac{d^{2} f_{q_{i}}}{d t_{n}^{2}}
\end{aligned}
$$

\section{B. Smoothness, cyclicity and vector of parameters}

The study is focused on a cyclical gait defined by an impact and SSP. We assume that the left foot is on support during the SSP, also $q_{0}=0$. The unknown vector $q_{i}$ is now for $i=$ $\{1 \ldots 6\}$. Following the hypotheses that are posed for the gait:

- The mobile foot is flat on the ground at the beginning and at the end of the step

- $\quad$ The angles of the trunk and the mobile foot are $T$ periodic

- The angles of the tibiae and the thighs are $2 T$-periodic

With the spline function, we consider the continuity and the smoothness of the function at the half-period. These conditions for the mobile foot, the trunk, the tibiae and the thighs lead to:

$$
\begin{array}{r}
a_{5_{0}}=b_{5_{0}}=0, a_{5_{2}}=b_{5_{2}}, a_{5_{3}}=b_{5_{3}}=-\frac{4}{3} a_{5_{2}} \\
a_{6_{0}}=b_{6_{0}}, a_{6_{2}}=b_{6_{2}}, a_{6_{3}}=b_{6_{3}}=-\frac{4}{3} a_{6_{2}} \\
a_{1_{0}}=b_{4_{0}}, a_{1_{2}}=b_{4_{2}}=a_{4_{2}}+6\left(a_{4_{0}}-a_{1_{0}}\right), \\
b_{1_{3}}=-6\left(\left(a_{1_{0}}-a_{4_{0}}\right)+\frac{4}{3}\left(a_{1_{1}}-a_{1_{2}}\right)\right) \\
a_{4_{0}}=b_{1_{0}}, a_{4_{2}}=b_{1_{2}}=a_{1_{2}}+6\left(a_{1_{0}}-a_{4_{0}}\right), \\
b_{4_{3}}=-6\left(\left(a_{4_{0}}-a_{1_{0}}\right)+\frac{4}{3}\left(a_{4_{1}}-a_{4_{2}}\right)\right) \\
a_{2_{0}}=b_{3_{0}}, a_{2_{2}}=b_{3_{2}}=a_{3_{2}}+6\left(a_{3_{0}}-a_{2_{0}}\right), \\
b_{2_{3}}=-6\left(\left(a_{2_{0}}-a_{3_{0}}\right)+\frac{4}{3}\left(a_{2_{1}}-a_{2_{2}}\right)\right) \\
a_{3_{0}}=b_{2_{0}}, a_{3_{2}}=b_{2_{2}}=a_{2_{2}}+6\left(a_{2_{0}}-a_{3_{0}}\right), \\
b_{3_{3}}=-6\left(\left(a_{3_{0}}-a_{2_{0}}\right)+\frac{4}{3}\left(a_{3_{1}}-a_{3_{2}}\right)\right)
\end{array}
$$

The impact model imposes a relation between the speed before and after the impact time for all mobile bodies. For example, the speed of the right thigh after the impact depends on (21) and on the speed of the left thigh just before the impact. These conditions are expressed as:

$$
\begin{aligned}
& a_{5_{1}}=b_{5_{1}}+\Delta_{5} \\
& a_{6_{1}}=b_{6_{1}}+\Delta_{6} \\
& a_{2_{1}}=b_{3_{1}}+\Delta_{2} \\
& a_{3_{1}}=b_{2_{1}}+\Delta_{3} \\
& a_{1_{1}}=b_{4_{1}}+\Delta_{1} \\
& a_{4_{1}}=b_{1_{1}}+\Delta_{4}
\end{aligned}
$$

In resume, the vector of parameters is $a_{i_{0}}$ for $i=$ $\{1,2,3,4,6\}, b_{j_{1}}$ and $a_{j_{2}}$ for $j=\{1 \ldots 6\}$. The total number of parameters for the trajectories is 17 .

Finally, the parameters $a_{i_{0}}$ which give the initial angular position could be found with the inverse geometric model of the Cartesian position of the hip $\left(x_{H}(0), z_{H}(0)\right)$. The period $T$ is added as parameter for the gait determination. The parameter's vector $\mathbf{p}=\left[x_{H}(0), z_{H}(0), a_{6_{0}}, b_{j_{1}}, a_{j_{2}}, T\right]$ is used in the following optimization process.

\section{OPTIMIZATION PROBLEM}

The research of optimal parameters to produce the best gait trajectories is challenging. The goal is to search trajectories minimizing a criterion representing the energetic consumption of the robots while respecting the constraints due to the biped environment. A gait is considered optimal if the gait is physically feasible and with the minimal of power supply.

We have also to solve a nonlinear minimizing problem under constraints that can be expressed:

$$
\begin{gathered}
\min _{\mathbf{p}} C_{\Gamma}(\mathbf{p}) \\
\text { under } \Psi(\mathbf{p}) \geq 0
\end{gathered}
$$

with $\Psi=\left[\Psi_{1} \Psi_{2} \Psi_{3} \Psi_{4} \Psi_{5} \Psi_{6} \Psi_{7}\right]^{T}$ are the constraints imposed by the initial configuration of the robot and the physical constraints considered before. The constraints are:

- $\Psi_{1}=F_{z}$ define the vector of the force along the z-axis and must be positive,

- $\quad \Psi_{2}=x_{Z M P}+l_{p}$ and $\Psi_{3}=-x_{Z M P}+\left(L_{p}-l_{p}\right)$ represent the limit position of the ZMP on $\mathrm{x}$-axis (ZMP must stay in the foot support) to guaranty the stability of the robot,

- $\Psi_{4}=q_{2}-q_{1}$ and $\Psi_{5}=q_{3}-q_{4}$ are the choice made to keep a gait human-like with no bend backward of knees,

- $\Psi_{6}=z_{H 2} \operatorname{resp} .\left(z_{H^{\prime} 2}\right)$ and $\Psi_{7}=z_{T 2} \operatorname{resp} .\left(z_{T^{\prime} 2}\right)$ are used to have the $\mathrm{z}$-coordinates of heel and toes of the mobile foot above the ground during the SSP.

The $\mathrm{x}$-coordinates of ZMP is calculated by:

$$
x_{Z M P}=\frac{\Gamma_{1}-h_{p} F_{x}-m_{p} s_{x} g}{F_{z}}
$$

The criterion used is the estimation of energy losses. It is defined by (40).

$$
C(\mathbf{p})=\frac{2}{d} \int_{0}^{T} k J \Gamma^{T} \Gamma+C s|\dot{\theta}|+F v \dot{\theta}^{2} d t
$$


with $\Gamma$ calculated from (17), $k J$ represents the matrix of Joules quality factor. $|\dot{\theta}|=\left[\left|\dot{\theta}_{i}\right|\right]$ for $i=\{1 \ldots 6\}$.

We propose to solve this problem by using the NelderMead simplex algorithm, see [11]. This algorithm solves nonlinear problem without constraints. The constraints are also added in the criterion (40) as Lagrange multiplier. The equation becomes:

$$
C_{E}(\mathbf{p})=C(\mathbf{p})+\frac{2}{d} \int_{0}^{T}\left(K \sum_{i=1}^{7}\left(e^{\left(\left|\Psi_{i}\right|-\Psi_{i}\right)}-1\right)\right) d t+E r r
$$

with $\Psi_{i}$ represents the constraints already defined, $K$ is the multiplier of Lagrange equal to $10^{6}$ in our calculation and $\mathrm{Err}$ can handle the errors due to the inverse geometric model. At the end of the optimization, we verify that all constraints are positive and Err is equal to zero in all cases.

\section{SIMULATIONS AND RESULTS}

The simulations were done with the geometrical and dynamic parameters of HYDROID Robot. The height of this biped robot is $1.39 \mathrm{~m}$ with a total mass of $45.36 \mathrm{~kg}$. The table II in appendix gives the physical parameters of each body part. For these simulations, the radii $r_{1}$ and $r_{2}$ are chosen equal to $5 \mathrm{~cm}$. The simulation objective is to compare the configurations defined in II and to find the best solution. Two series of optimization were performed, one with the matrix of Coulomb friction $C s$ equal to zero and the other with actuator specific values given in table III in appendix. We will compare the effects of this matrix on the evolution of criteria and will observe the joint torque profile. We will examine the estimated cost of gait between our robot and those of the literature studies [15] for the human and other robots.

In Fig. 3, the evolution of optimal energetic criteria versus the walking speed is presented. For the first set of simulation with zero Coulomb friction, we see that the energy criteria of RK robot are lower than those of CK robot. The energy gain is about $4.6 \%$ in average. Considering the Coulomb friction of the actuators, the criterion of the configuration with rolling knee (RK) is reduced by $4.4 \%$ in average compared to the configuration with revolute joints (CK). Furthermore, we obtain a ratio of 6 between the energetic criteria with the null friction coefficients and those of actuators, for the slow speeds and a ratio of 2 for the high speeds.

TABLE I. ESTIMATED COST OF TRANSPORT $c_{e t}$

\begin{tabular}{|c|c|}
\hline Biped Robot : & $c_{e t}$ \\
\hline Asimo & 3.2 \\
\hline Collins' robot & 0.2 \\
\hline Robot CK $\left(C s=0_{6 \times 6}\right)$ & 0.295 \\
\hline Robot CK $\left(C s \neq 0_{6 \times 6}\right)$ & 0.6 \\
\hline Robot RK $\left(C s=0_{6 \times 6}\right)$ & 0.292 \\
\hline Robot RK $\left(C s \neq 0_{6 \times 6}\right)$ & 0.58 \\
\hline Human : & 0.2 \\
\hline
\end{tabular}

Fig. 4 shows the stick diagram of both robots of $1 \mathrm{~m} / \mathrm{s}$ without friction coefficient. The biped robots have the same gait. For the RK robot, the mobile foot is a bit higher on the start and the trunk more leaned forward at the end of the step. Fig. 5 presents the evolution of torques for the walking speed of $1 \mathrm{~m} / \mathrm{s}$. Without friction, the torques of the mobile

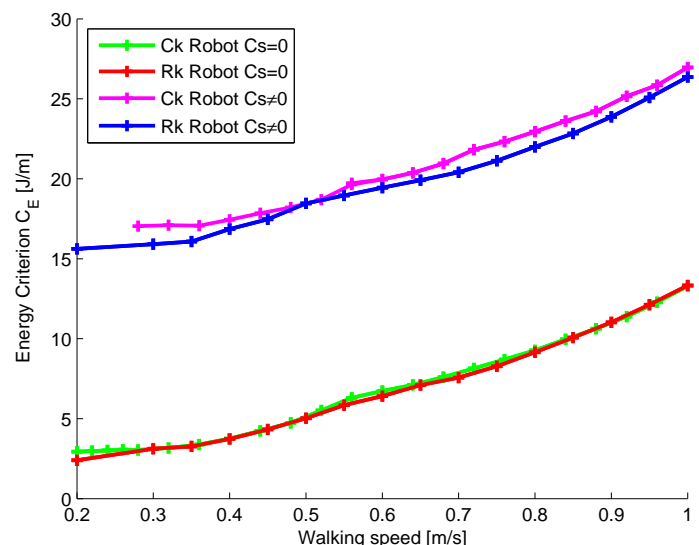

Fig. 3. Evolution of optimal energy criterion in function of the walking speed

hip for the RK robot are reduced in beginning of the step and the period of step is increased. With the Coulomb frictions, the hip torques of the two legs of RK Robot are lower than those of the CK robot. The torques of the RK robot knees are higher. The rolling knee on support leg accepts more torque and reduces the torques on the hip joints in all cases.
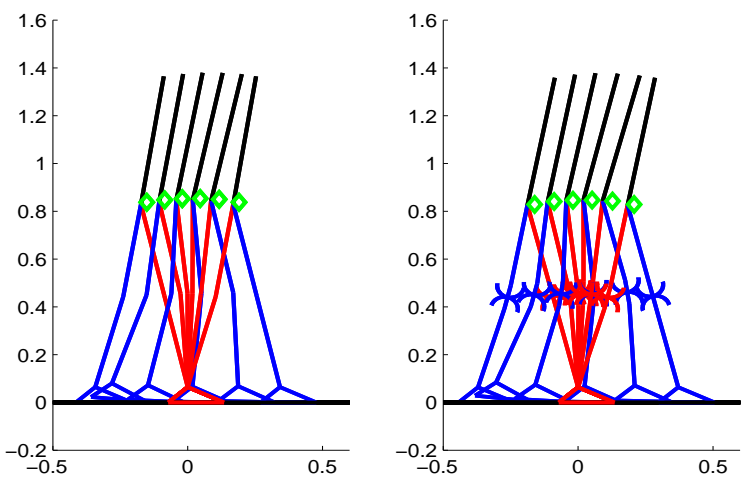

Fig. 4. Stick diagrams of the biped with the two configurations for the walking speed for $1 \mathrm{~m} / \mathrm{s}$. On the left, the CK robot and on the right, the RK robot. The green markers represent the center of mass of biped

The table I shows the cost of transport $c_{e t}$ calculated by the (energyused $) /($ weight $) *($ distancetraveled $)$. We can see that the results of the robot without friction coefficient are near the $c_{e t}$ of the human.

\section{CONCLUSIONS}

The model of the rolling knee joint robot has been developed. Our simulation program computes the joint torques and the forces on the feet for the different gait. The trajectories are parametrized by cubic spline functions and have allowed the resolution of the energy optimization problem. Without friction coefficients, the optimization process shows that the rolling knee structure gives an energy reduction of $4.6 \%$ in average. We demonstrate that the energy consumption of robot with zero Coulomb coefficients gives results near of human energy consumption. Taking into account the mechanical friction, the 

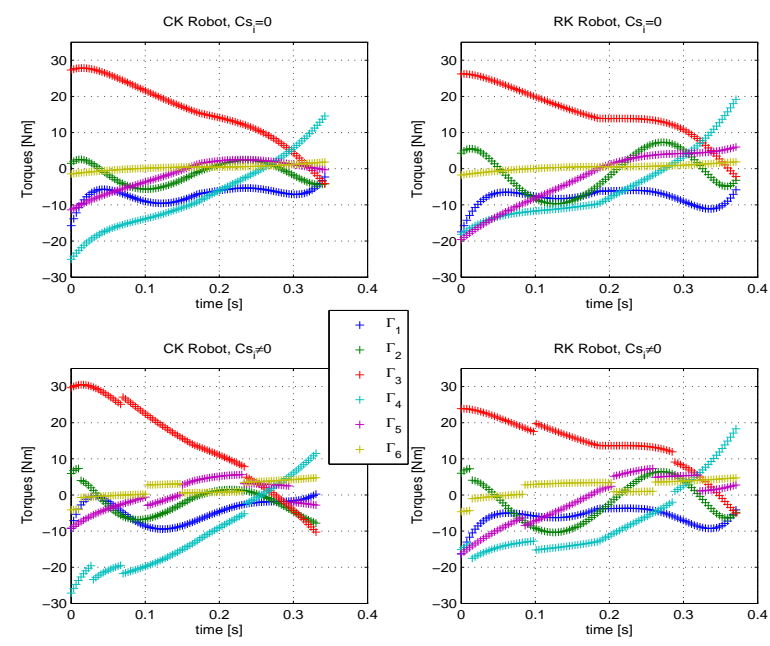

Fig. 5. Evolution of torques for the walking speed at $1 \mathrm{~m} / \mathrm{s}$

energy criterion is increased by a factor of 2 to 5 depending on the walking speed. The structure with rolling knees reduces slightly the energy criterion but especially facilitates the robot design to integrate linear actuators and transmissions that reduce the friction terms.

In future, we will develop other gaits such as Double Support Phases (DSP) including foot rotation. The influence of the radii $r_{1}$ and $r_{2}$ represents another interesting challenge for the robot design. For the selection of linear motor or artificial muscles, the design with two actuators locations, one on the thigh, the other on the trunk, may be considered. A more accurate model of friction can then be initiated.

\section{ACKNOWLEDGMENTS}

The authors gratefully acknowledge the contribution of French National Research Agency under the project number ANR-09-SEGI-011-R2A2.

\section{REFERENCES}

[1] A. Hamon and Y. Aoustin, "Cross four-bar linkage for the knees of a planar bipedal robot," in 10th IEEE-RAS International Conference on Humanoid Robots, Humanoids 2010, 2010, pp. 379-384.

[2] G. Van Oort, R. Carloni, D. Borgerink, and S. Stramigioli, "An energy efficient knee locking mechanism for a dynamically walking robot," in Proceedings - IEEE International Conference on Robotics and Automation, 2011, pp. 2003-2008.

[3] M. Hobon, N. Lakbakbi Elyaaqoubi, and G. Abba, "Quasi Optimal Gait of a Biped Robot with a Rolling Knee Kinematic," in IFAC 18th World Congress 2011, Milano, Italy, 2011, pp. 11580-11 587.

[4] C. Chevallereau and Y. Aoustin, "Optimal reference trajectories for walking and running of a biped robot," Robotica, vol. 19, no. 5, pp. 557-569, 2001.

[5] P.-B. Wieber, "Constrained dynamics and parametrized control in biped walking," in International Symposium on Mathematical Theory of Networks and Systems, Perpignan, France, 2000.

[6] E. R. Westervelt, J. Grizzle, and D. E. Koditschek, "Hybrid zero dynamics of planar biped walkers," IEEE Transactions on Automatic Control, vol. 48, pp. 42-56, 2001.

[7] G. Gini, U. Scarfogliero, and M. Folgheraiter, "Human-oriented biped robot design: Insights into the development of a truly anthropomorphic leg," in Proceedings - IEEE International Conference on Robotics and Automation, 2007, pp. 2910-2915.
[8] G. Bessonnet and P. Sardain, "Optimal dynamics of actuated kinematic chains. part 1: Dynamic modelling and symbolic differentiations," European Journal of Mechanics, A/Solids, vol. 24, no. 3, pp. 452-471, 2005.

[9] M. Scheint, M. Sobotka, and M. Buss, "Compliance in gait synthesis: Effects on energy and gait," in 2008 8th IEEE-RAS International Conference on Humanoid Robots, Humanoids 2008, 2008, pp. 259264.

[10] G. Cabodevila and G. Abba, "Rank selection genetic algorithm to determine quasi optimal constrained gait for a biped robot," in Proc. Int. Conf. on Climbing and Walking Robots, B. eds., Ed., Brussels, Belgium, Nov. 1998, pp. 303-310.

[11] J. C. Lagarias, J. A. Reeds, M. H. Wright, and P. E. Wright, "Convergence properties of the nelder-mead simplex method in low dimensions," SIAM J. Optim., vol. 9, pp. 112-147, 1998.

[12] C. Oatis, Kinesiology: The Mechanics and Pathomechanics of Human Movement. Lippincott Williams \& Wilkins, 2004.

[13] M. Flannery, S. Flanagan, E. Jones, and C. Birkinshaw, "Compliant layer knee bearings. part i: Friction and lubrication," Wear, vol. 269, no. $5-6$, pp. $325-330,2010$

[14] S. Collins, M. Wisse, and A. Ruina, "A three-dimensional passivedynamic walking robot with two legs and knees," International Journal of Robotics Research, vol. 20, no. 7, pp. 607-615, 2001.

[15] S. H. Collins and A. Ruina, "A bipedal walking robot with efficient and human-like gait," in Proceedings - IEEE International Conference on Robotics and Automation, vol. 2005, 2005, pp. 1983-1988.

[16] B. Armstrong, "Friction: Experimental Determination, Modeling and Compensation," in Proceedings - IEEE International Conference on Robotics and Automation, Philadelphia, PN, USA, April 24-29, 1988, vol. 3, pp. 1422-71988.

[17] G. Abba and P. Sardain, "Modeling of frictions in the transmission elements of a robot axis for its identification," in IFAC Proceedings Volumes (IFAC-PapersOnline), vol. 16, 2005, pp. 7-12.

[18] M. Spong and M. Vidyasagar, Robot dynamics and control, J. Wiley and Sons, Eds. New-York: John Wiley and Sons, 1991.

[19] W. Khalil and E. Dombre, Modeling, identification and control of robots, T. . Francis, Ed., Bristol, PA, 2002.

[20] F. Pfeiffer and C. Glocker, Multibody Dynamics with Unilateral Contacts. New York: Wiley, 1996.

[21] Y. Banno, Y. Harata, K. Taji, and Y. Uno, "Optimal trajectory design for parametric excitation walking," in 2009 IEEE/RSJ International Conference on Intelligent Robots and Systems, IROS 2009, 2009, pp. 3202-3207.

\section{APPENDIX}

TABLE II. PARAMETERS OF HYDROID ROBOT

\begin{tabular}{|c||c||c||c||c|}
\hline Body & Lengths & Masses & $\begin{array}{c}\text { Inertia } \\
\text { moments } \\
{\left[\mathrm{kg} . \mathrm{m}^{2}\right]}\end{array}$ & $\begin{array}{c}\text { Positions } \\
\text { of CoM } \\
{[\mathrm{m}]}\end{array}$ \\
\hline Feet & $\begin{array}{c}L_{p}=0.207 \\
l_{p}=0.072 \\
h_{p}=0.064\end{array}$ & 0.678 & 0.001 & $\begin{array}{c}s x=0.013 \\
s z=0.032\end{array}$ \\
\hline Tibia & 0.392 & 2.188 & 0.028 & 0.168 \\
\hline Thigh & 0.392 & 5.025 & 0.066 & 0.168 \\
\hline Trunk & 0.543 & 29.270 & 0.81 & 0.192 \\
\hline
\end{tabular}

TABLE III. PARAMETERS OF ACTUATOR

\begin{tabular}{|c||c||c||c|}
\hline Actuator & $\begin{array}{c}\mathrm{kJ} \\
{\left[\mathrm{J} / \mathrm{Nm}^{2}\right]}\end{array}$ & $\begin{array}{c}\mathrm{Cs} \\
{[\mathrm{Nm}]}\end{array}$ & $\begin{array}{c}\mathrm{Fv} \\
{[\mathrm{Nms}]}\end{array}$ \\
\hline Ankle & $k J_{1}=0.016$ & $c s_{1}=1.608$ & $f v_{1}=0.367$ \\
\hline Knee & $k J_{2}=0.039$ & $c s_{2}=1.260$ & $f v_{2}=0.207$ \\
\hline Hip & $k J_{3}=0.010$ & $c s_{3}=2.204$ & $f v_{3}=0.902$ \\
\hline
\end{tabular}

